\title{
PERISTALTIC PUMPING OF ELLIS FLUID THROUGH A FLEXIBLE TUBE WITH COMPLETE SLIP EFFECTS
}

\section{R. Saravana ${ }^{1, a}$, S. Sreenadh ${ }^{2}$, P. Rajesh Kumar ${ }^{3}$ and V. Ramesh Babu ${ }^{4}$}

${ }^{1}$ Department of Mathematics, Madanapalle Institute of Technology \& Science, Madanapalle 517 325, India, asaravanasvu@gmail.com

${ }^{2}$ Department of Mathematics, Sri Venkateswara University, Tirupati 517 502, India, profsreenadh@gmail.com

${ }^{3}$ Research Scholar, Department of Mathematics, Rayalaseema University, Kurnool 518 002, India, prajeshreddy1@gmail.com

${ }^{4}$ Department of Mathematics, National Sanskrit University, Tirupati 517 507, India, drvrbabu45@gmail.com

\begin{abstract}
:
The paper investigates the role of complete velocity slip on the axisymmetric peristaltic transport of Ellis fluid through a uniform flexible tube considering the long wavelength and tiny Reynolds number assumptions. The equations governing the axisymmetric flow are solved and provided the analytical expressions for axial velocity, stream function, pressure difference for shear thickening and shear thinning fluids and the effect of emerging parameters on the pressure rise with time averaged flux and stream line patterns have been discussed graphically. The trapping phenomenon results showed that the size of trapping bolus enhances with increasing values of first and second slip parameters.
\end{abstract}

Keywords: Ellis fluid, complete slip, shear thickening behavior, shear thinning behavior.

\section{NOMENCLATURE}

\begin{tabular}{|c|c|c|c|}
\hline$a$ & Radius of the circular tube & $q$ & Volume flow rate in wave frame \\
\hline$c$ & Speed of the wave & $Q(z, t)$ & Volume flow rate in Laboratory frame \\
\hline$b$ & Amplitude & $t$ & time \\
\hline$(R, \theta, Z)$ & $\begin{array}{l}\text { Coordinates of circular tube in Laboratory } \\
\text { frame of reference }\end{array}$ & \multicolumn{2}{|c|}{ Greek symbols } \\
\hline$(r, \theta, z)$ & $\begin{array}{l}\text { Coordinates of circular tube in wave frame } \\
\text { of reference }\end{array}$ & $\lambda$ & Wave length \\
\hline$\psi$ & Stream function in wave frame reference & $\beta_{1}$ & First slip parameter \\
\hline$\Psi$ & $\begin{array}{l}\text { Stream function in laboratory frame } \\
\text { reference }\end{array}$ & $\beta_{2}$ & Second slip parameter \\
\hline$p$ & Pressure & $\eta_{1}$ & Ellis parameter \\
\hline$w$ & $\begin{array}{l}\text { Velocity component in wave frame of } \\
\text { reference }\end{array}$ & $\tau_{r z}$ & extra stress tensor component \\
\hline$n$ & Adjustable parameter & $\eta_{0}$ & zero shear viscosity \\
\hline
\end{tabular}

\section{Introduction}

Peristalsis is an important transport phenomenon characterized by the contraction and relaxation of flexible tube and naturally observed in tubular organs of human body to transport the fluids. Peristaltic flow is an essential mechanism for fluid transport in tubular organs for example, the oesophagus, the gastrointestinal tract, the ureter and the urethra. Recent detailed exposition on the peristalsis can be found in research of (Saravana et al.,2018, Hayat et al., 2019, Manjunatha et al., 2019, Noreen and Kousar, 2019, Akram et al., 2019). 
The peristaltic propulsion is also a novel and mechanical displacement method to transfer the liquid within a flexible tube. In rotor peristaltic mechanism, when the rotor turns, the tube under compression closes forcing the fluid being pumped to move through the tube. As the tube opens and returns to its starting state after passing the fluid and further the flow is induced to pump. The action also helps to propel the fluid without the contamination of outside environment. It is built to pump various fluid types in different marine structures or vessels, industrial and in medical environments. The mechanism is found in marine uses connected to pumping of slurry and in emptying of bilges and tanks of the boats and other vessels.

The transporting liquid includes water, abrasive or corrosive, shear thickening agents such as gels and oxidizers. Shear thinning agents such as paints and polymer solutions. The behavior of flow which mimics the shearthinning fluid and/or shear thickening fluid is Power-law model. When the deviations from the model are significantly demonstrated at low shear rates, it is appropriate to use Ellis model. The limiting value behavior of power-law fluid model under consideration, Ellis proposed the three parameter fluid model to exhibit the flow characteristics over a small shear rate ranges by swapping the roles of shear stress and strain rates.

The flow of an Ellis fluid through a circular tube with an impermeable wall have been explored by Longwell (1966). Ali et al. (2015) reported the peristaltic transport of non-Newtonian Ellis fluid through a channel. Abbas et al. (2017) demonstrated the peristaltic blood of Ellis fluid through a non-uniform channel with compliant walls. Recently, Kumar et al. (2018) addressed the peristaltic pumping of an Ellis fluid model in an inclined tube with wall properties. Ali et al. (2019) studied the electro-osmotic peristaltic flow of an Ellis fluid in contact with a Newtonian fluid through an axisymmetric tube.

Motivated by the influence of above works, we investigated the peristaltic transport of an Ellis fluid in a circular tube with the modelling of complete wall slip under small values of Reynolds number. The velocity field, stream function, volume flow rate and pressure rise over one wavelength are determined. The effects of Ellis parameter $\eta_{1}$ and the two slip parameters on the pumping characteristics and stream line patterns are depicted graphically.

\section{Mathematical Formulation}

The Ellis equation model is explored under the consideration of work (Ali et al., 2019) and Rathy (1976) as

$\eta_{0} \tau_{r z}+\eta_{1}\left|\tau_{r z}\right|^{n-1} \tau_{r z}=\frac{d w}{d r}$

The model has three adjustable set of positive parameters namely the zero shear viscosity $\eta_{0}$, and $\eta_{1}$ and $n$. When suitably chosen $n$ greater than unity, the model approaches Newtonian fluid for small values of extra stress tensor component $\tau_{r z}$. On the other hand, when $n$ is subjected to less than unity, Newtonian model approaches for large values of $\tau_{r z}$. The model is highly flexible and recovers the Newtonian fluid model as $\eta_{1} \rightarrow 0$ and the power-law model as $\eta_{0} \rightarrow 0$.

We consider the peristaltic flow associated with an incompressible Ellis fluid through a circular tube of radius $a$ (see Fig. 1) in presence of complete wall slip. The axisymmetric inertia free flow is generated by the sinusoidal wave train propagation with wave speed ' $c$ ' along the tube in the cylindrical geometry.

The geometry of the wall surface is defined as

$$
R=H(Z, t)=a+b \sin \frac{2 \pi}{\lambda}(Z-c t)
$$

where $b$ refers the amplitude, $\lambda$ refers wave length. 


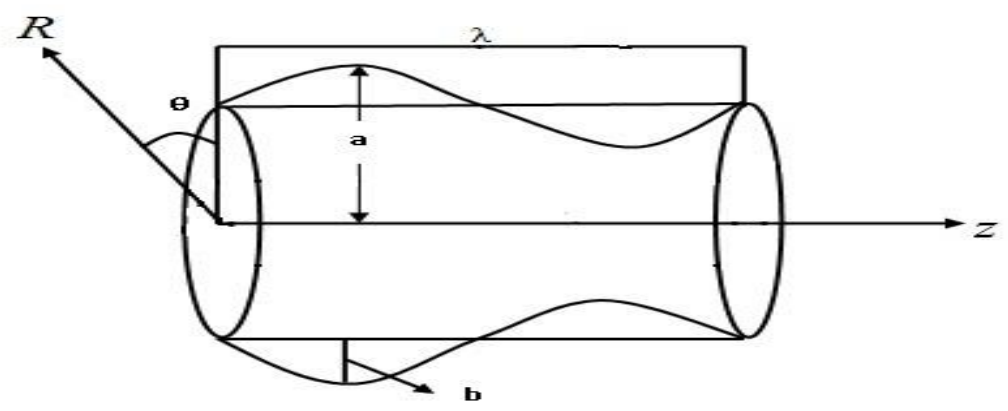

Fig. 1. Physical model

Such a flow rises unsteady in the laboratory frame $(R, \theta, Z)$ and attain steady in the wave frame $(r, \theta, z)$. The transformation between the lab frame and wave frame of reference leads to the following

$r=R, \theta=\theta, z=Z-c t, \psi=\Psi-\frac{R^{2}}{2}$ and $p(Z, t)=p(z)$

where $\psi$ and $\Psi$ denotes the wave and the laboratory frame stream functions respectively.

Further, we define the following dimensionless quantities.

$\bar{h}=\frac{H}{a} ; \phi=\frac{b}{a} ; \bar{w}=\frac{w}{c} ; \bar{r}=\frac{r}{a} ; \bar{z}=\frac{z}{\lambda} ; \bar{t}=\frac{c t}{\lambda} ; \bar{\psi}=\frac{\psi}{\pi a^{2} c} ; \bar{w}=\frac{1}{\bar{r}} \frac{\partial \bar{\psi}}{\partial \bar{r}} ; \bar{u}=-\frac{1}{\bar{r}} \frac{\partial \bar{\psi}}{\partial \bar{r}} ; \bar{\tau}_{r z}=\frac{\eta_{0} a \tau_{r z}}{c} ;$

$\bar{p}=\frac{\eta_{0} a^{2} p}{\pi c \lambda} ; \bar{Q}=\frac{Q}{\pi a^{2} c} ; \bar{\eta}_{1}=\frac{\eta_{1} c^{n-1}}{\eta_{0}^{n} a^{n-1}} ; \beta_{1}=\frac{\alpha_{1}}{a} ; \beta_{2}=\frac{\alpha_{2}}{a^{2}}$

where $\bar{w}$ is the axial velocity component in the wave frame, $\beta_{1}$ is the first slip parameter and $\beta_{2}$ is the second slip parameter.

Under the lubrication approach and using the above non-dimensional quantities, we arrive the equation of motion as (omitting the bars):

$\frac{1}{r} \frac{\partial}{\partial r}\left(r \tau_{r z}\right)=\frac{\partial p}{\partial z}$

$\frac{d w}{d r}=\tau_{r z}+\eta_{1}\left|\tau_{r z}\right|^{n-1} \tau_{r z}$

The appropriate boundary conditions for the concern problem of study are

$\tau_{r z}$ is finite at $r=0$

$w=-1-\beta_{1} \frac{\partial w}{\partial r}-\beta_{2} \frac{\partial^{2} w}{\partial r^{2}} \quad$ at $r=h$

$\psi=0$ at $\quad r=0$

When the flow takes place past a rough surface, it is well known that the usual no slip condition is not valid at the surface $r=h(z)$. The slip at such rough surfaces has been remarked through several slip conditions such as Navier (1827), Beavars and Joseph (1967), Saffman (1971), Vajravelu et al. (2013), Saravana et al. (2017) and Prasad and Yasa (2020). In view of the flow on such surfaces, the improved boundary condition Eq. (8) proposed by Ebaid et al. (2017) is introduced at the bounding wall incorporating the complete slip.

\section{Solution Procedure}

Simplifying the Eq. (5) and Eq. (6) by imposing the boundary conditions Eq. (7) and Eq. (8) leads to the existence of velocity field as

$w=-1-P\left(\frac{h^{2}}{4}+\frac{\beta_{2}}{2}+\frac{\beta_{1} h}{2}-\frac{r^{2}}{4}\right)-P^{n}\left(\frac{\eta_{1} h^{n+1}}{2^{n}(n+1)}+\beta_{2} \frac{\eta_{1} n h^{n}}{2^{n}}+\beta_{1} \frac{\eta_{1} h^{n}}{2^{n}}-\frac{\eta_{1} r^{n+1}}{2^{n}(n+1)}\right)$

in which $P=\frac{\partial p}{\partial z}$ 
Integrating the Eq. (10) by applying the condition (9) leads to the stream function as

$\psi=-P^{n}\left(\frac{\eta_{1} h^{n+1} r^{2}}{2^{n+1}(n+1)}+\frac{\beta_{2} \eta_{1} n h^{n-1} r^{2}}{2^{n+1}}+\frac{\beta_{1} \eta_{1} h^{n} r^{2}}{2^{n+1}}-\frac{\eta_{1} r^{n+3}}{2^{n}(n+1)(n+3)}\right)-P\left(\frac{h^{2} r^{2}}{8}+\frac{\beta_{2} r^{2}}{4}+\frac{\beta_{1} h r^{2}}{4}-\frac{r^{4}}{16}\right)-\frac{r^{2}}{2}$

The rate of volume flow $q$ through each cross section in the wave frame is presented by

$$
\begin{aligned}
q & =2 \int_{0}^{h} w r d r \\
& =-h^{2}-P\left(\frac{h^{4}}{8}+\frac{\beta_{2} h^{2}}{2}+\frac{\beta_{1} h^{3}}{2}\right)-\frac{P^{n}}{2^{n}}\left(\frac{\eta_{1} h^{n+3}}{n+3}+\beta_{2} \eta_{1} n h^{n+1}+\beta_{1} \eta_{1} h^{n+2}\right)
\end{aligned}
$$

The instantaneous volume flow rate $Q(z, t)$ in the laboratory frame is given by

$$
\begin{aligned}
Q(z, t) & =2 \int_{0}^{h}(w+1) r d r \\
& =q+h^{2} \\
& =P\left(\frac{h^{4}}{8}+\frac{\beta_{2} h^{2}}{2}+\frac{\beta_{1} h^{3}}{2}\right)-\frac{P^{n}}{2^{n}}\left(\frac{\eta_{1} h^{n+3}}{n+3}+\beta_{2} \eta_{1} n h^{n+1}+\beta_{1} \eta_{1} h^{n+2}\right)
\end{aligned}
$$

Averaging Eq. (13) over one period gives the time-averaged volume flow rate $\bar{Q}$ is followed by

$$
\begin{gathered}
\bar{Q}=\frac{2}{T} \int_{0}^{T} \int_{0}^{h}(w+1) r d r d t \\
=q+\frac{1}{T} \int_{0}^{T} h^{2} d t \\
=q+1+\frac{\phi^{2}}{2}
\end{gathered}
$$

Choosing Ellis parameter $\eta_{1}=0$ in Eq. (13), the corresponding results are identical to the peristaltic motion of an incompressible Newtonian fluid in a flexible tube with complete slip mentioned below as

$$
\frac{d p}{d z}=-\frac{\left(q+h^{2}\right)}{\frac{h^{4}}{8}+\frac{\beta_{2} h^{2}}{2}+\frac{\beta_{1} h^{3}}{2}}
$$

Further, by considering $\beta_{2}=0$ in the Eq. (15), we have the pressure gradient for the peristaltic pumping of Newtonian fluid in a circular tube with complete slip as

$$
\frac{d p}{d z}=\frac{-\left(q+h^{2}\right)}{\frac{h^{4}}{8}+\frac{\beta_{1} h^{3}}{2}}
$$

Employing $\beta_{1}=\beta_{2}=0$ in the Eq. (15), we find that

$$
\frac{d p}{d z}=\frac{-8\left(q+h^{2}\right)}{h^{4}}
$$

The result deducted agree with the corresponding result of Jaffrin and Shapiro (1971) for the peristaltic transport of an incompressible Newtonian fluid in a tube

Eq. (13) permits a nonlinear relationship in pressure gradient, thus the expression examined independently for $n<1$ and $n>1$ which corresponds to shear thickening and shear thinning fluids respectively. 
We now turn to the pumping characteristics for particular set of non-negative values of $n$, say, $n=\frac{1}{2}$ and 2 are respectively to shear thickening and shear thinning fluids.

In particular, if one considers shear thickening value $n=\frac{1}{2}$, then pumping characteristics is explored as

$$
\begin{aligned}
\frac{d p_{1}}{d z}= & \frac{-\left(\frac{784}{h^{4}}\left(q+h^{2}\right)\left(1+\frac{4 \beta_{2}}{h^{2}}+\frac{4 \beta_{1}}{h}\right)-\frac{128}{h} \eta_{1}^{2}\left(1+\frac{7 \beta_{2}}{4 h^{2}}+\frac{7 \beta_{1}}{2 h}\right)^{2}\right)}{98\left(1+\frac{4 \beta_{2}}{h^{2}}+\frac{4 \beta_{1}}{h}\right)} \pm \\
& \frac{\sqrt{\left(\frac{784}{h^{4}}\left(q+h^{2}\right)\left(1+\frac{4 \beta_{2}}{h^{2}}+\frac{4 \beta_{1}}{h}\right)-\frac{128}{h} \eta_{1}^{2}\left(1+\frac{7 \beta_{2}}{4 h^{2}}+\frac{7 \beta_{1}}{2 h}\right)^{2}\right)^{2}-4\left(49\left(1+\frac{4 \beta_{2}}{h^{2}}+\frac{4 \beta_{1}}{h}\right)^{2}\right)\left(\frac{56}{h^{4}}\left(q+h^{2}\right)\right)^{2}}}{98\left(1+\frac{4 \beta_{2}}{h^{2}}+\frac{4 \beta_{1}}{h}\right)}
\end{aligned}
$$

If the respective shear thinning value is $n=2$, then the pumping characteristics is given by

$$
\frac{d p_{2}}{d z}=\frac{-5 h^{4}\left(1+\frac{4 \beta_{2}}{h^{2}}+\frac{4 \beta_{1}}{h}\right) \pm \sqrt{\left(5 h^{4}\left(1+\frac{4 \beta_{2}}{h^{2}}+\frac{4 \beta_{1}}{h}\right)\right)^{2}-320 \eta_{1} h^{5}\left(1+\frac{2 \beta_{2}}{h^{2}}+\frac{\beta_{1}}{h}\right)\left(q+h^{2}\right)}}{4 \eta_{1} h^{5}\left(1+\frac{2 \beta_{2}}{h^{2}}+\frac{\beta_{1}}{h}\right)}
$$

Eq. (18) is integrated with respect to $z$ per wave length for obtaining Pressure rise $\Delta p$ across one wavelength for shear thickening fluid as

$$
\Delta P_{1}=\int_{0}^{1} \frac{d p_{1}}{d z} d z
$$

Eq. (19) is integrated with respect to $z$ per wave length for obtaining Pressure rise $\Delta p$ across one wavelength for shear thinning fluid as

$$
\Delta P_{2}=\int_{0}^{1} \frac{d p_{2}}{d z} d z
$$

\section{Results and Discusion}

The effect of Ellis parameter $\eta_{1}$, first slip parameter $\beta_{1}$ and second slip parameter $\beta_{2}$ on the pressure rise with time-averaged flux $\bar{Q}$ for shear thickening and shear thinning fluids are analyzed and depicted graphically. From Eq. (20), we have explored the pressure rise $\Delta P_{1}$ for a shear thickening fluid as a function of timeaveraged flux $\bar{Q}$. Fig. 2 depicts the pressure rise $\Delta P_{1}$ variation of Ellis parameter $\eta_{1}$ on shear thickening fluid with time-averaged flux. We notice that $\Delta P_{1}$ has been enhanced with the growth of Ellis parameter. The curve $\eta_{1}=0$ corresponds to Newtonian fluid with complete slip. Figs. 3 and 4 illustrate the impact of the two slip parameters on the variation of $\Delta P_{1}$ with time-averaged flux. We see that the pressure rise gradually increases with increasing first slip parameter $\beta_{1}$ and second slip parameter $\beta_{2}$. The curve $\beta_{2}=0$ in Fig. 4 represents the peristaltic flow of Ellis fluid (shear thickening) with partial slip. The greater the second slip parameter the more the pressure rise against which the pump works for a shear thickening fluid.

From Eq. (21), we have explored the pressure difference $\Delta P_{2}$ for a shear thinning fluid as a function of time averaged flux $\bar{Q}$. Figs. 5-7 are plotted to observe the distribution of pressure rise across the tube with $\bar{Q}$ for 
different values of $\eta_{1}, \beta_{1}$ and $\beta_{2}$. Fig. 5 indicates $\Delta P_{2}$ with $\bar{Q}$ for small values of Ellis parameter. We found that increasing $\eta_{1}$ decreases the pumping performance in pumping region $(\Delta P>0)$ and the pumping curves coincides in free pumping $(\Delta P=0)$ and co-pumping region $(\Delta P<0)$. Figs. 6 and 7 illustrate the effect of the slip parameters on the variation of $\Delta P_{2}$ with time-averaged flux. It is found that increasing first slip parameter $\beta_{1}$ and second slip parameter $\beta_{2}$ decreases the pumping and free pumping. The curve $\beta_{2}=0$ in Fig. 7 represents the peristaltic flow of Ellis fluid (shear thinning) with partial slip.

The streamline patterns are illustrated from Eq. (11). The effects of Ellis fluid parameter $\eta_{1}$ corresponding to shear thinning fluid are depicted in Fig. 8(a-c). We found that the trapping bolus size decreases with the enhancement of $\eta_{1}$. The effects of power-law index parameter $n$ on trapping phenomenon are demonstrated in Fig. 9(a-c). From these figures, we found that the volume of the trapped bolus decreases when the fluid changes from shear thickening to shear thinning. There is also a greater number of trapped boluses occur for the Newtonian fluid $(n=1)$. The influence of slip parameters on stream line patterns are visualized in Figs. 10 and 11. It is evident that the size of trapping bolus strengthens with increasing first and second slip parameters.

\subsection{Illustrations}

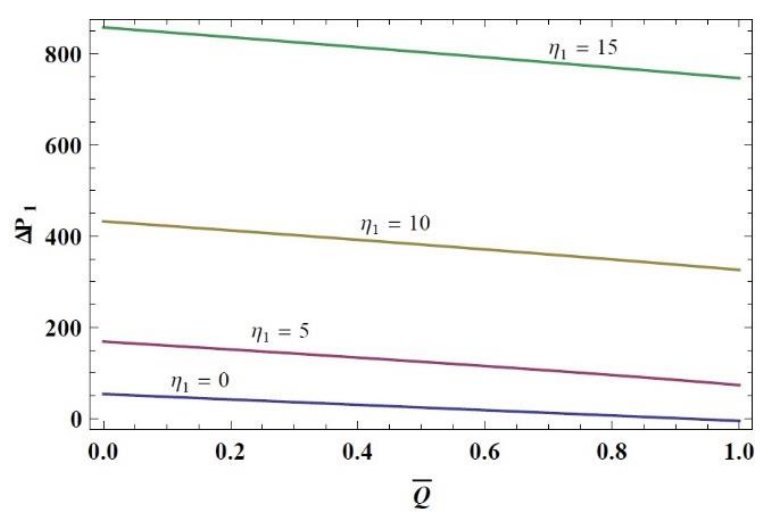

Fig. 2. The distribution of $\Delta P_{1}$ with $\bar{Q}$ for differen

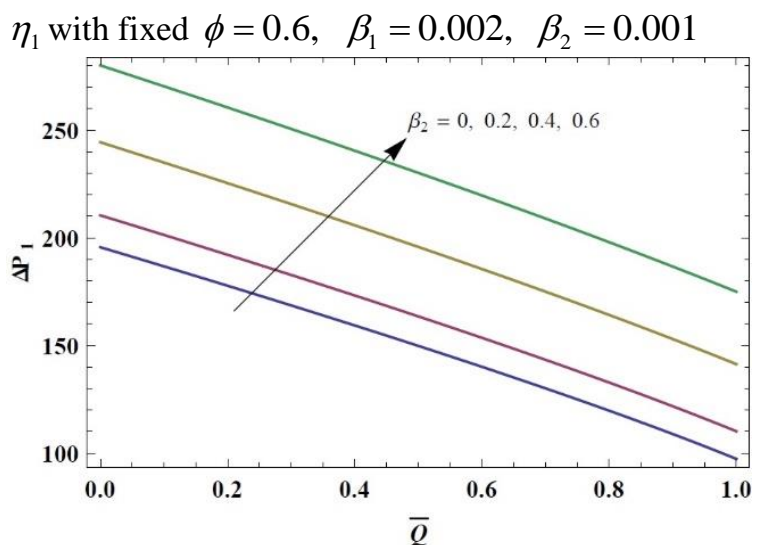

Fig. 4. The distribution of $\Delta P_{1}$ with $\bar{Q}$ for different $\beta_{2}$ with fixed $\phi=0.6, \eta_{1}=5, \beta_{1}=0.02$

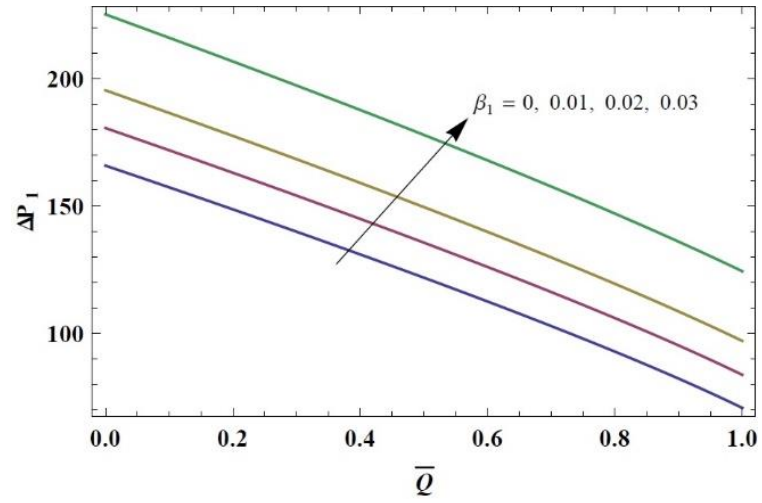

Fig. 3. The distribution of $\Delta P_{1}$ with $\bar{Q}$ for different $\beta_{1}$ with fixed $\phi=0.6, \eta_{1}=5, \beta_{2}=0.001$

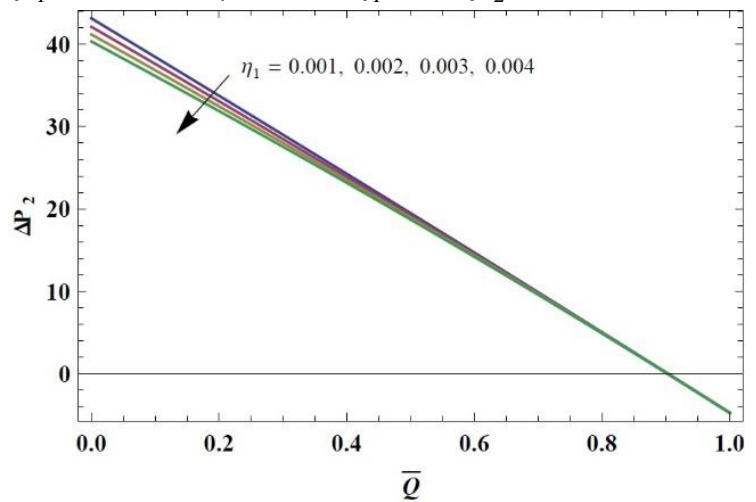

Fig. 5. The distribution of $\Delta P_{2}$ with $\bar{Q}$ for different $\eta_{1}$ with fixed $\phi=0.6, \beta_{1}=0.001, \beta_{2}=0.1$ 
R. Saravana, S. Sreenadh, P. Rajes Kumar and V. Ramesh Babul /Journal of Naval Architecture and Marine Engineering, 17(2020) 79-88
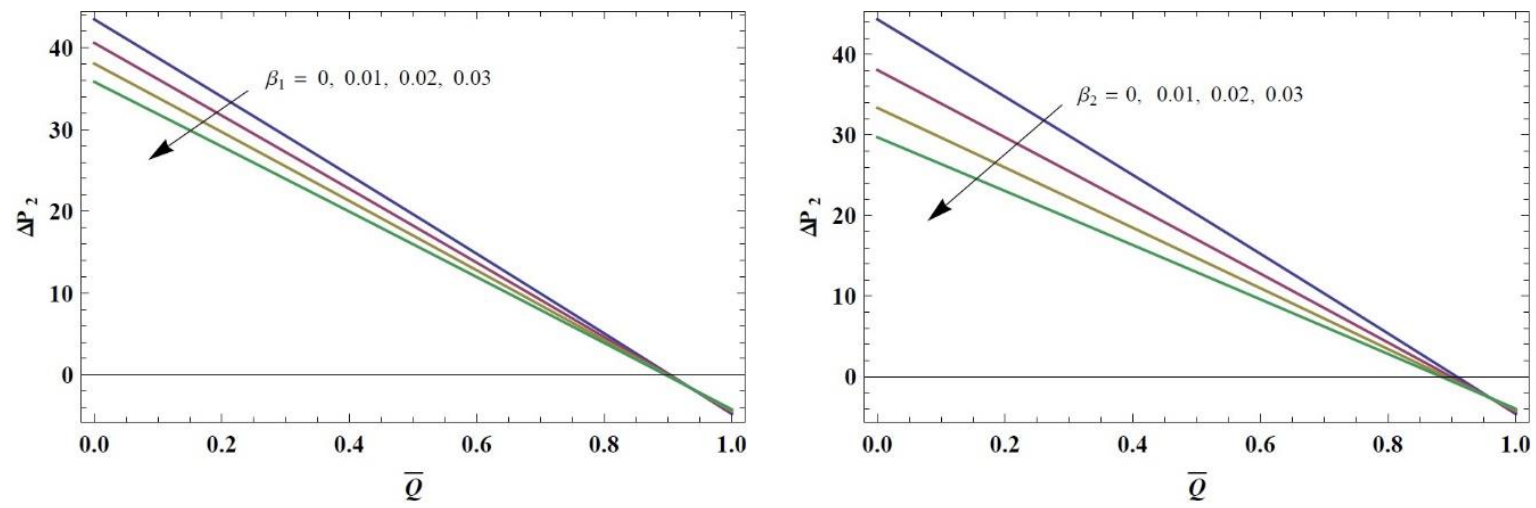

Fig. 6. The distribution of $\Delta P_{2}$ with $\bar{Q}$ for different Fig. 7. The distribution of $\Delta P_{2}$ with $\bar{Q}$ for different $\beta_{1}$ with fixed $\phi=0.6, \eta_{1}=0.001, \beta_{2}=0.01$ $\beta_{2}$ with fixed $\phi=0.6, \eta_{1}=0.001, \beta_{1}=0.02$

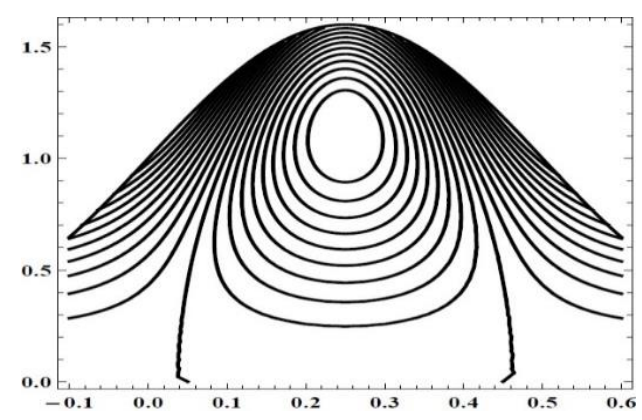

(a)

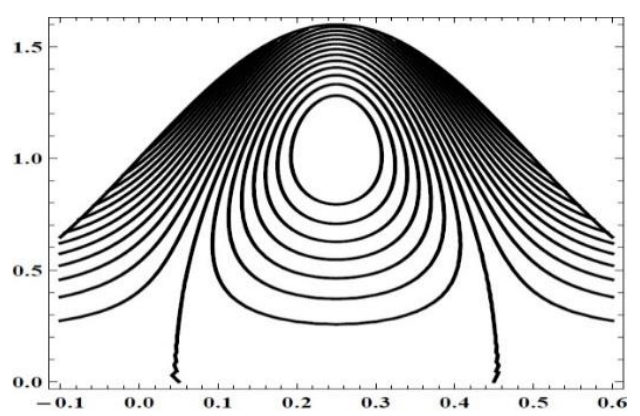

(b)

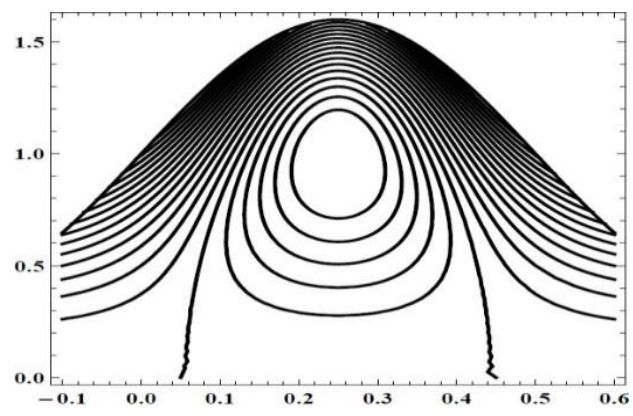

(c)

Fig. 8. Stream line demonstration for (a) $\eta_{1}=0.01$ (b) $\eta_{1}=0.05$ (c) $\eta_{1}=0.1$ with fixed $\phi=0.6, P=-3$, $\beta_{1}=0.01, \beta_{2}=0.01, n=2$

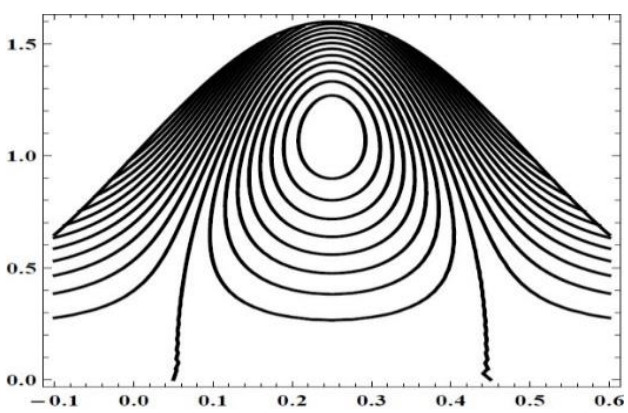

(a)

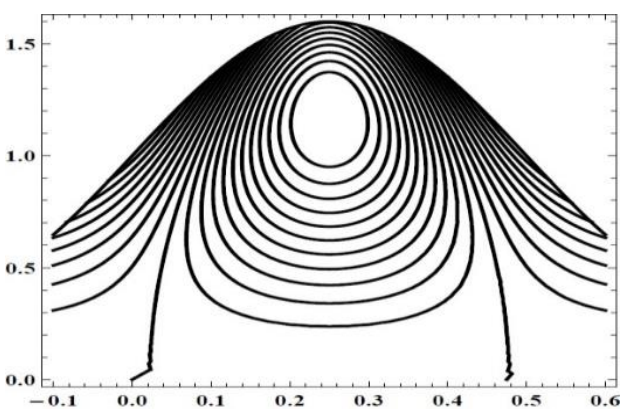

(b)

Peristaltic pumping of Ellis fluid through a flexible tube with complete slip effects

85 


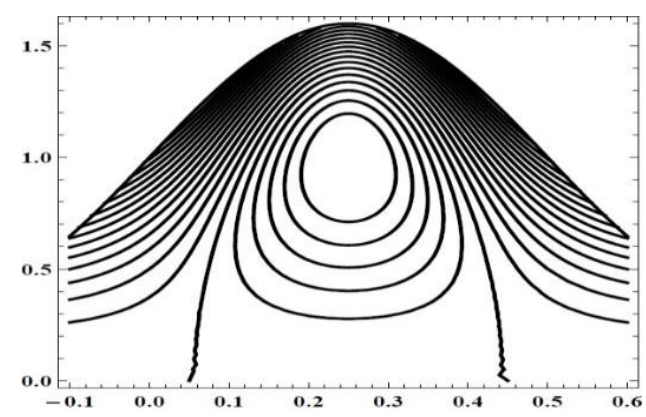

(c)

Fig. 9. Stream line demonstration for (a) $n=0.001$ (b) $n=1$ (c) $n=2$ with fixed $\phi=0.6, P=-3$, $\beta_{1}=0.01, \beta_{2}=0.01, \eta_{1}=0.01$

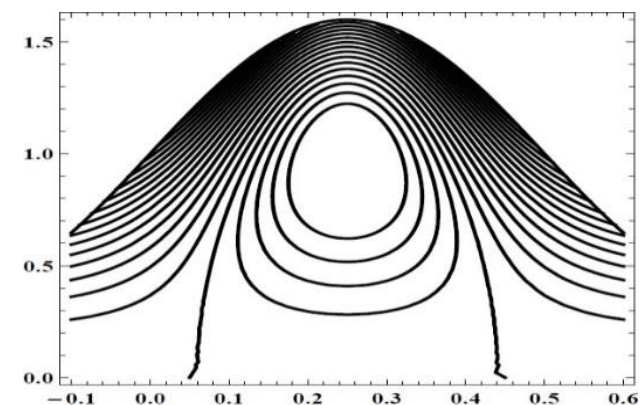

(a)

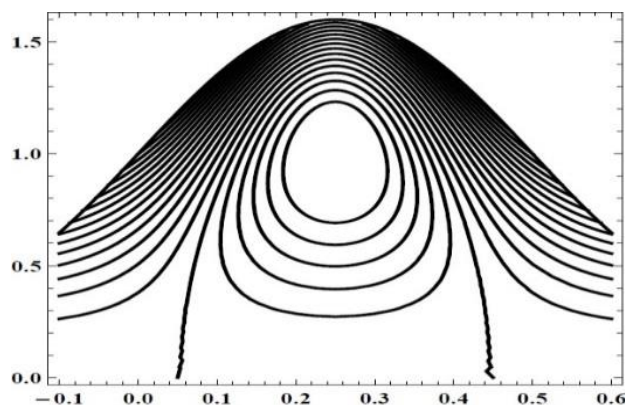

(b)

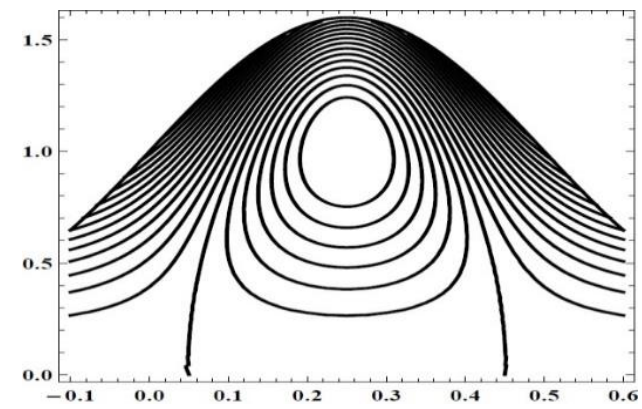

(c)

Fig. 10. Stream line demonstration for (a) $\beta_{1}=0.00$ (b) $\beta_{1}=0.02$ (c) $\beta_{1}=0.04$ with fixed $\phi=0.6$, $P=-3, n=2, \beta_{2}=0.01, \eta_{1}=0.1$

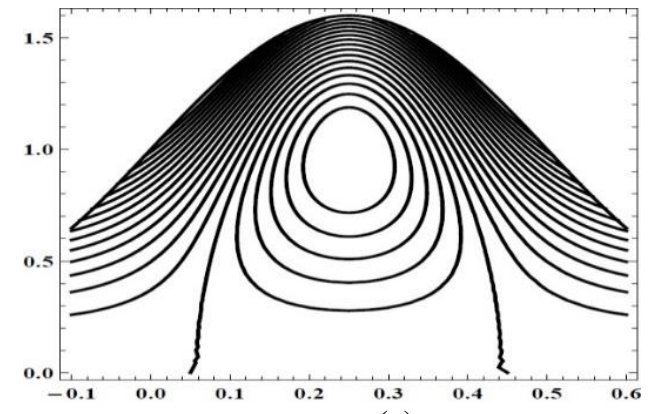

(a)

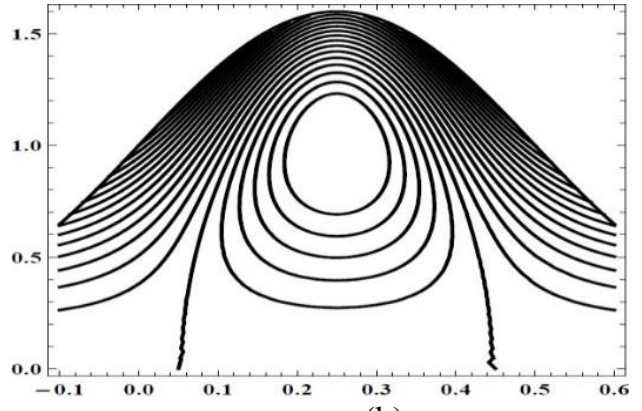

(b) 


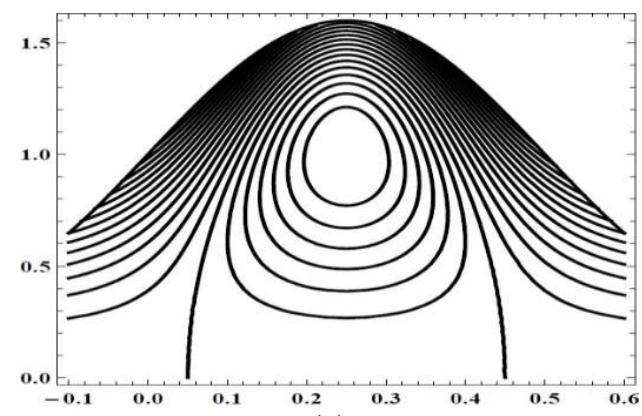

(c)

Fig. 11. Stream line demonstration for (a) $\beta_{2}=0.00$ (b) $\beta_{2}=0.01$ (c) $\beta_{2}=0.02$ with fixed $\phi=0.6$, $P=-3, n=2, \beta_{1}=0.02, \eta_{1}=0.1$

\section{Conclusions}

In this paper, we demonstrated the task of incorporating the complete velocity slip conditions at the uniform tube walls of the peristaltic flow of an Ellis fluid under the constraint of long wavelength and small Reynolds number. The interesting findings are as follows

$>$ Pressure rise for shear thickening fluid increases with large values of Ellis parameter.

> Pressure rise for shear thickening fluid increases with the growth of first slip parameter and second slip parameter.

$>$ Increasing Ellis parameter decreases the pumping performance in pumping region and the pumping curves coincides in free pumping and co-pumping region.

$>$ The size of trapping bolus enhances with growth of first and second slip parameters.

$>$ The trapping bolus size declines with large Ellis fluid parameter variation.

\section{References}

Abbas, A.M., Bhatti, M.M. and Rashidi, M.M. (2017): Peristaltic blood flow of Ellis fluid through a nonuniform channel having compliant Walls, Journal of Nanofluids, Vol. 6, No. 2, pp. 318-323. https://doi.org/10.1166/jon.2017.1314.

Akram, S., Aly, E. H., Afzal, F. and Nadeem, S. (2019): Effect of the Variable Viscosity on the Peristaltic Flow of Newtonian Fluid Coated with Magnetic Field: Application of Adomian Decomposition Method for Endoscope, Coatings, Vol. 9, No. 8, Article Id: 524. https://doi.org/10.3390/coatings9080524.

Ali, N., Abbasi, A. and Ahmad, I. (2015): Channel flow of Ellis fluid due to peristalsis, AIP Advances, Vol. 5, No. 9, Article ID: 097214, pp. 1-9. https://doi.org/10.1063/1.4932042.

Ali, N., Hussain, S., Ullah, K and Beg, O.A. (2019): Mathematical modelling of two-fluid electro-osmotic peristaltic pumping of an Ellis fluid in an axisymmetric tube, European Physical Journal Plus, Vol. 134 (2019) 141 https://doi.org/10.1140/epjp/i2019-12488-2.

Beavers. G.S. and Joseph, D.D. (1967): Boundary conditions at a naturally permeable wall, J. Fluid Mech., 30 (1967) 197-206. https://doi.org/10.1017/S0022112067001375.

Ebaid, A., Aly, E. H. and Vajravelu, K. (2017): Analytical Solution for Peristaltic Transport of Viscous Nano fluid in an Asymmetric Channel with Full Slip and Convective Conditions, Communications in Theoretical Physics. Vol. 68, No. 3, pp. 96-102. https://doi.org/10.1088/0253-6102/68/1/96.

Hayat, T., Aslam, N., Khan, M. I., Khan, M. I. and Alsaedi, A. (2019): MHD peristaltic motion of JohnsonSegalman fluid in an inclined channel subject to radiative flux and convective boundary conditions. Computer Methods and Programs in Biomedicine. Vol. 180, 2019, 104999. https://doi.org/10.1016/j.cmpb.2019.104999.

Jaffrin, M.Y. and Shapiro, A.H. (1971): Peristaltic pumping, Annual Review of Fluid Mechanics, Vol. 3, pp. 13 36. https://doi.org/10.1146/annurev.fl.03.010171.000305

Kumar, K. T., Kavitha, A., Saravana, R. (2018): Peristaltic flow of an Ellis fluid model in an inclined uniform tube with wall properties, International Journal of Mechanical Engineering and Technology, Vol. 9, No. 2, pp. $15-27$.

Longwell, P.A. (1966): Mechanics of fluid flow, McGraw Hill Book Company, New York. 
Manjunatha, G., Rajashekhar C., Vaidya, H., Prasad K.V. and Viharika J.U. (2019): Influence of convective conditions on the peristaltic mechanism of power-law fluid through a slippery elastic porous tube with different waveforms, Multidiscipline Modeling in Materials and Structures. Vol. 16, No. 2, pp. 340-359. https://doi.org/10.1108/MMMS-01-2019-0006.

Navier, C.L.M.H. (1827): Sur les lois du mouvement des fluides, Mémoires de l'Académie Royale des Sciences de l'Institut de France, Vol. 6, pp. 389-440.

Noreen, S. and Kousar, T. (2019): Hall, ion slip and ohmic heating effects in thermally active sinusoidal channel, Propulsion and Power Research, Vol. 8, No. 3, pp. 263-273. https://doi.org/10.1016/j.jppr.2019.02.002.

Prasad, K. M. and Yasa, P. (2020). Flow of non-Newtonian fluid through a permeable artery having nonuniform cross section with multiple stenosis. Journal of Naval Architecture and Marine Engineering, 17(1), 3138. https://doi.org/10.3329/jname.v17i1.40942.

Rathy, R.K. (1976): An Introduction to Fluid Dynamics, Oxford and IBH Publishing Co., New Delhi.

Saffman, P.G. (1971): On the Boundary Conditions at the Surface of a Porous Medium. Studies in Applied Mathematics, Vol. 50, No. 2, pp. 93-101. https://doi.org/10.1002/sapm197150293.

Saravana, R., Hemadri Reddy, R., Suresh Goud, J and Sreenadh, S. (2017): MHD peristaltic flow of a hyperbolic tangent fluid in a nonuniform channel with heat and mass transfer, IOP Conf. Series: Materials Science and Engineering, Vol. 263, No. 6, Article ID: 062006. doi:10.1088/1757-899X/263/6/062006.

Saravana, R., Vajravelu, K. and Sreenadh, S. (2018): Influence of Compliant Walls and Heat Transfer on the Peristaltic Transport of a Rabinowitsch Fluid in an Inclined Channel. Zeitschrift für Naturforschung A, Vol. 73, No. 9, pp. 833-843. https://doi.org/10.1515/zna-2018-0181.

Vajravelu, K., Sreenadh, S. and Saravana, R. (2013): Combined influence of velocity slip, temperature and concentration jump conditions on MHD peristaltic transport of a Carreau fluid in a non-uniform channel, Applied Mathematics and Computation, Vol. 225, pp. 656-676. https://doi.org/10.1016/j.amc.2013.10.014. 